Ann. Biol. anim. Bioch. Biophys., r967, 7 (I), 47-58.

\title{
ÉVACUATION STOMACALE COMPAREE DU LAIT ET DU YOGHOURT CHEZ LE RAT
}

\author{
R. ROZEN \\ avec la collaboration technique de W. LoIseL \\ Laboratoire d'Études des Qualités biologiques des Aliments de l'Homme, \\ Centre national de Recherches zootechniques, 78-Jouy-en-Josas
}

\section{SOMIMAIRE}

Des rats adultes reçoivent, par intubation gastrique ou par ingestion, l'un ou l'autre des produits suivants :

- lait à I $30 \mathrm{~g}$ de matière sèche et Io $\mathrm{g}$ de matière grasse par litre,

- yogourt, obtenu à partir du lait précédent par action de ferments lactiques sélectionnés.

Les animaux sont sacrifiés à divers intervalles de temps, et on détermine les quantités de matière sèche présentes dans le contenu de l'estomac.

$\mathbf{I}^{\circ}$ Le lait quitte l'estomac avec une rapidité remarquable, quel que soit le mode d'administration : environ $25 \mathrm{p}$. Ioo de la quantité donnée sont évacués au cours des premières minutes, $5^{\circ} \mathrm{p}$. I00 dans la première demi-heure, $75 \mathrm{p}$. Ioo dans la I $^{\mathrm{re}}$ heure.

Après ingestion, la relation qui lie la quantité de matière sèche présente dans le contenu de l'estomac ( $y$, en $\mathrm{mg}$ ) au temps écoulé depuis l'ingestion ( $t$, en minutes) est, durant les 3 premières heures, une fonction semi-logarithmique :

$$
y=-\mathrm{I} 77,4 \log t+448 .
$$

$2^{\mathbf{0}}$ Le yogourt est évacué de l'estomac un peu plus lentement que le lait, lorsqu'ils sont donnés par intubation.

Dans le cas de l'ingestion, l'évacuation du yogourt ne se fait pas suivant une loi simple. Elle est d'abord plus lente que celle du lait : I heure après l'ingestion, il reste dans l'estomac $36 \mathrm{p}$. Ioo de la quantité de matière séche initiale du yogourt (au lieu de 24 p. 1oo pour le lait). Puis elle se fait au même rythme que celle du lait. Enfin, elle s'accélère et l'évacuation gastrique du yogourt se termine plus tôt que celle du lait.

\section{INTRODUCTION}

Les connaissances sur le transit des différents types de lait dans le tube digestif sont peu avancées et peu précises.

Les cliniciens et les physiologistes se sont attachés surtout à comparer les modalités de la digestion du lait de vache et du lait maternel chez le jeune enfant : on sait que le premier donne dans l'estomac un coagulum ferme et compact, moins facilement attaqué par le suc gastrique que le coagulum diffluent formé par le lait de 
femme. Ce phénomène, joint au pouvoir neutralisant très marqué du lait de vache, explique pour une large part que l'évacuation pylorique de ce lait soit plus lente que celle du lait maternel. Différents traitements ont été proposés pour obvier à ces inconvénients, en particulier les traitements thermiques - qui abaissent la "tension " du coagulum -, l'homogénéisation, le passage sur permutite en vue d'abaisser le taux du calcium par échange d'ions, etc.

Quelques auteurs ont tenté récemment de préciser ces notions, en étudiant l'évacuation gastrique de divers types de laits. Leurs observations, résumées dans le tableau I, sont malheureusement peu nombreuses.

Ainsi, dans la discussion du travail où ils montrent que l'évacuation gastrique du yogourt est plus lente que celle du lait. BALzoLA et al. (Ig6I) écrivent : "Nous n'avons découvert dans l'immense littérature concernant le yoghourt aucune donnée précise quant à la rapidité de l'évacuation gastrique - et SoMOGyI (I960) confirme cette absence - depuis les travaux de ToBLER datant de Igo6. En " marquant " le yoghourt par de la baryte, ou en effectuant des prélèvements fractionnés, cet auteur trouve que les laits fermentés quittent plus tôt l'estomac, et après Von NooRDEN (1) tout le monde répète cette affirmation. ")

Il nous a donc paru intéressant d'effectuer une étude comparée de l'évacuation gastrique du lait et du yoghourt, en utilisant le Rat. Il est évident que, dans un tel domaine, l'expérimentation sur l'animal ne peut se substituer complètement aux observations sur 1'Homme : mais elle permet de recourir à des méthodes plus précises et peut donc fournir des indications précieuses pour l'orientation de nouvelles recherches sur 1'Homme.

\section{MISE AU POINT D'UNE MÉTHODE D'ÉTUDE}

Un essai préliminaire a été réalisé en vue de déterminer :

- les possibilités d'étude du transit digestif du lait par prélèvement, postmortem, du contenu de l'estomac et de l'intestin du Rat;

- les modalités d'application de la méthode.

\section{Technique}

L'essai porte sur 36 rats Wistar mâles pesant de 220 à $250 \mathrm{~g}$, soumis depuis le sevrage à un même régime alimentaire (biscuits " Extralabo ").

Après un jeûne de 24 heures, 6 d'entre eux sont sacrifiés par décapitation. Les 30 autres rats reçoivent, par intubation gastrique $\left(^{8}\right), 6 \mathrm{ml}$ de lait reconstitué à partir d'une poudre à $26 \mathrm{p}$. Ioo de matière grasse (soit $748 \mathrm{mg}$ de matière sèche) et porté à la température de $20^{\circ} \mathrm{C}$; ils sont partagés en 6 groupes de 5 sujets qu'on sacrifie respectivement 3 minutes, I heure, 2 heures, 4 heures et 8 heures après l'intubation.

(2) Von Noorden et Salomon (igro).

(2) Les stress provoqués par la décapitation et par l'intubation risquent évidemment d'influer sur la vidange de l'estomac. Il en est de même de toute méthode de sacrifice ; la décapitation a été choisie parce qu'elle provoque une mort rapide. Quant à l'intubation, elle permet de relever avec précision le moment de l'adininistration (instant zéro); ses effets seront comparés plus loin à ceux de l'ingestion. 


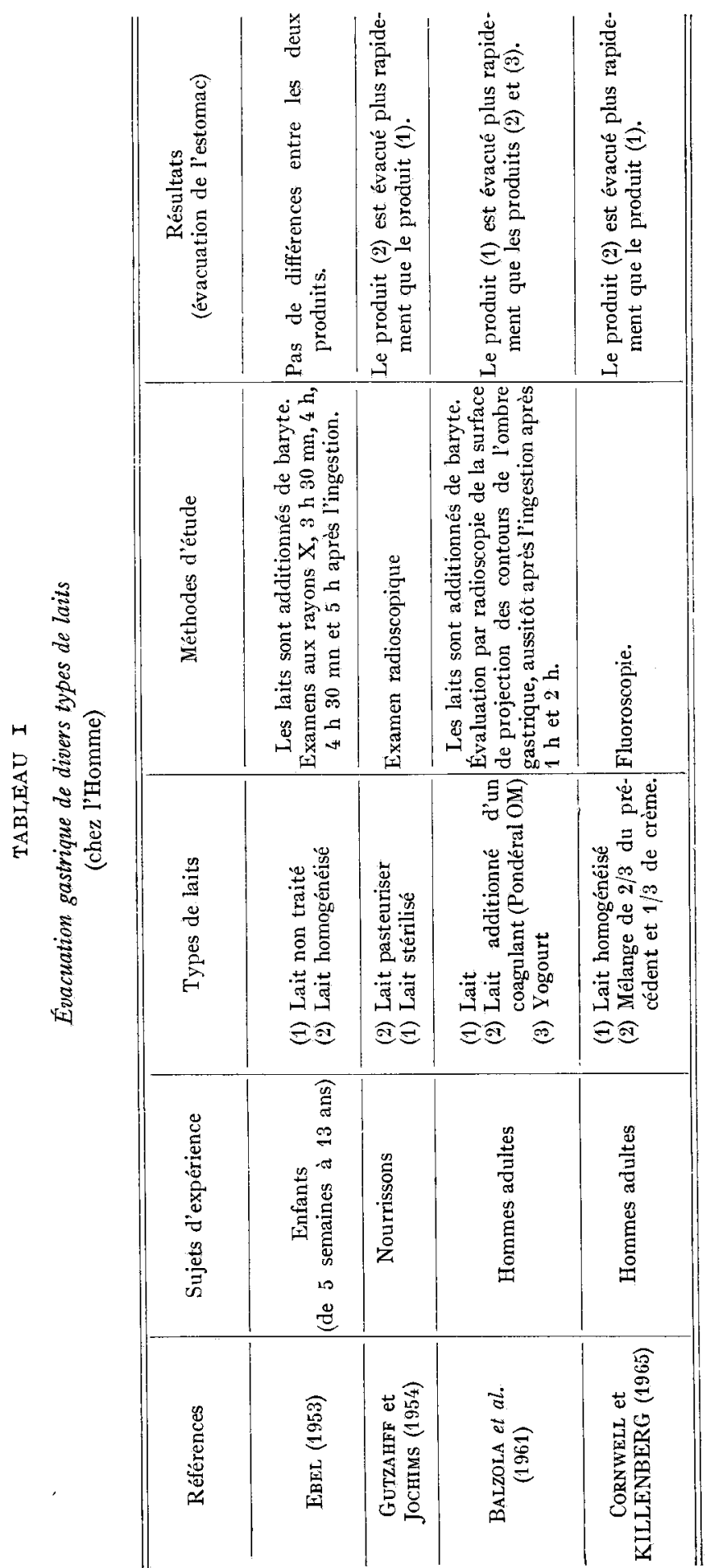

Annales de Biologie animale. - 1967 . 
Une minute après la décapitation de chaque animal, on pose des pinces de Kosher au niveau du cardia, du pylore, de la jonction duodéno-jejunale (visible très facilement chez le Rat) et avant le cæcum. On dégage le tube digestif, on le lave extérieurement à l'eau distillée et on recueille dans des boîtes tarées le contenu de l'estomac (incisé le long de la grande courbure), du duodénum et du reste du grêle, en rinçant abondamment (mais avec précaution) à l'eau distillée.

Les contenus digestifs (auxquels on joint les eaux de lavage) sont déshydratés à $75^{\circ}$ dans une étuve ventilée, jusqu’à poids constant.

\section{Résultats et conclusions}

Les résultats de l'essai sont réunis dans la figure I.

On voit que, chez les rats qui ont jeûné 24 heures, 1'estomac ne contient plus qu'une faible quantité de matière sèche. En revanche, cette quantité est encore élevée dans le grêle, duodénum compris (compte tenu de la faible capacité de ce dernier) (1).

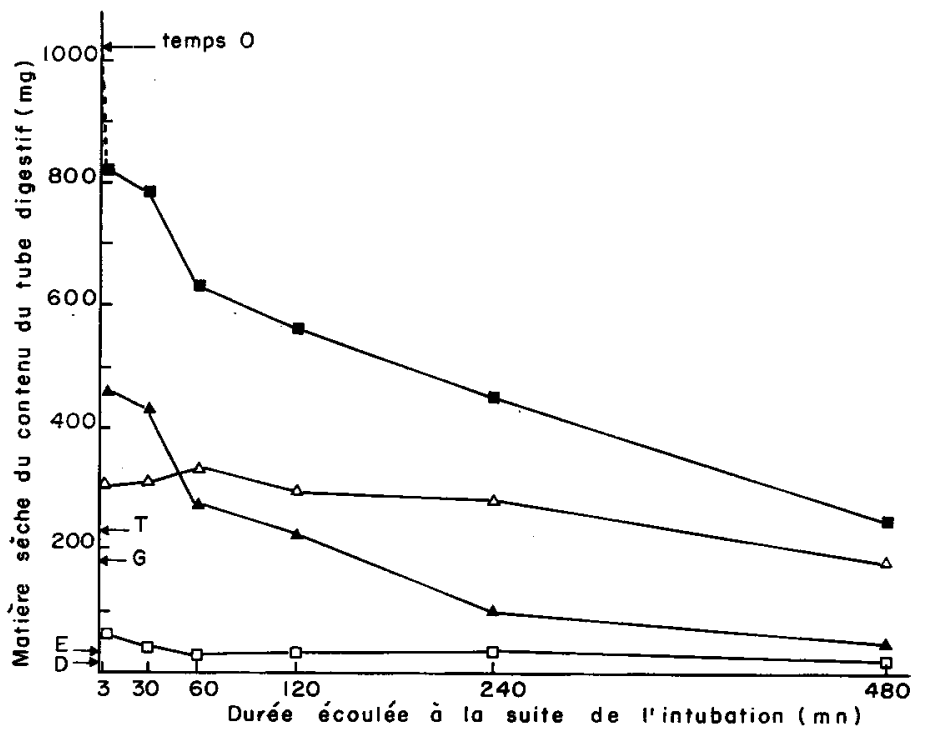

FIG. I. - Transit gastro-intestinal de la matière sèche après intubation de $6 \mathrm{ml}$ de lait

Les courbes représentent la matière sèche du contenu de l'estomac (triangles noirs), du duodénum (carrés blancs), du reste du grêle (triangles blancs) et de l'ensemble des trois segments (carrés noirs).

Les flèches portées le long de l'axe des ordonnées indiquent le " niveau de base " de la matière sèche dans l'estomac (E), le duodénum (D),

le reste du grèle (G) et le total des trois segments (T).

Chez les rats qui ont reçu du lait, l'évolution du taux de matière sèche au cours de la digestion est très différente au niveau des trois segments étudiés. L'évacuation de l'estomac, très rapide au début, devient de plus en plus lente avec le temps ; 8 heures après l'intubation, le taux de matière sèche est à peine supérieur au taux de base. Dans le duodénum, 3 minutes après l'intubation, le taux de la matière sèche

(1) Dans la suite du travail, le taux de matière sèche dosé dans le tube digestif du Rat à jeun depuis 24 heures sera appelé "taux de base ". Il est dû pour une part à la présence de substances endogènes, mais il s'explique aussi par la présence de résidus provenant de repas antérieurs, surtout dans l'intestin. 
est deux fois et demie plus élevé que le taux de base ; mais il s'abaisse vite et ne varie pratiquement plus pendant la fin de l'étude. Dans le reste du grêle, le taux de matière sèche est élevé dès la première détermination, mais il varie peu au cours des 4 premières heures ; après 8 heures, il correspond au taux de base.

D'autre part, il est intéressant de souligner que la variabilité individuelle des résultats est beaucoup plus importante au niveau du duodénum et du grèle qu'au niveau de l'estomac.

Il a paru possible de tirer de ces résultats quelques conclusions utiles pour la mise au point du protocole de l'étude expérimentale proprement dite.

Io L'étude post mortem de la cinétique de la vidange stomacale du lait paraît plus facile à interpréter que celle de son transit dans l'intestin.

Il a donc été décidé de n'étudier comparativement le transit digestif du lait et celui du yogourt qu'au niveau de l'estomac.

$2^{\circ}$ On peut se demander si la rapidité avec laquelle le lait passe dans le duodénum est normale, ou si elle est due aux conditions d'expériences (dose élevée, mode d'administration).

Quelques déterminations complémentaires réalisées sur des rats recevant par intubation $3 \mathrm{ml}$ de lait suggèrent effectivement que l'évacuation pylorique pendant les premières minutes est accélérée lorsque la dose de lait administrée est élevée (tabl. 2).

TABLEAU 2

Évacuation stomacale du lait après intubation de 3 ou $6 \mathrm{ml}$

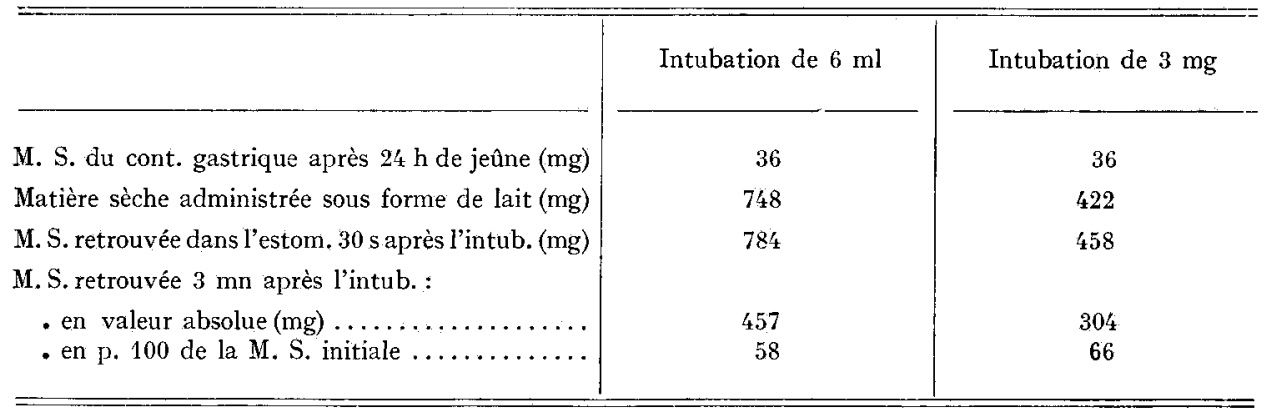

Il a donc été décidé de n'administrer que $3 \mathrm{ml}$ de lait ou de yogourt aux animaux dans l'étude expérimentale.

Quant au mode d'administration, il a paru intéressant de réaliser deux expériences :

- l'une, comportant l'intubation des produits étudiés, en raison de l'avantage que présente l'évaluation précise de 1' " instant zéro ";

- l'autre, comportant l'ingestion spontanée des mêmes produits, les animaux étant entraînés à consommer leur ration dans un délai aussi bref que possible.

$3^{\circ}$ Dans la dernière expérience - la plus intéressante - le nombre d'animaux à sacrifier a été fixé à 9 par point expérimental (au lieu de 5 dans l'étude préliminaire) 


\section{ÉTUDE DE L'ÉVACUATION DE L'ESTOMAC APRÈS ADMINISTRATION DE LAIT OU DE YOGHOURT}

PAR INTUBATION

\section{r. Protocole expérimental}

\section{a) Caractéristiques des produits étudiés.}

Le yogourt est généralement préparé en France à partir d'un lait privé d'une grande partie de ses matières grasses et renforcé en matière sèche par addition de lait écrémé en poudre. Pour en tenir compte, les deux produits administrés aux animaux ont été préparés dans les conditions suivantes :

-_ Le lait est obtenu en mélangeant en proportions convenables deux poudres " instantanées " préparées elles-mêmes à partir de lait exempt d'antibiotiques : l'une à 26 p. Ioo de matière grasse, l'autre à 0,5 p. 10o de matière grasse ( ${ }^{(1)}$. Le mélange est dilué avec la quantité d'eau nécessaire pour que le lait reconstitué contienne $13^{\circ} \mathrm{g}$ de matière sèche de ıo $\mathrm{g}$ de matière grasse par litre. Ce lait est ensuite chauffé à $85^{\circ} \mathrm{C}$ pendant $\mathrm{I}_{9}$ minutes $\left({ }^{2}\right)$. Son $\mathrm{pH}$ est en moyenne de $6,60\left(6,5^{2}\right.$ à 6,66$)$.

Une partie de ce lait est refroidie rapidement à $20^{\circ} \mathrm{C}$ et administrée au $\mathrm{I}^{\mathrm{er}}$ lot d'animaux.

Yoghourt. - L'autre partie du lait précédent sert à la préparation du yoghourt, selon le mode opératoire décrit par VASSAL. Le lait, maintenu à $45^{\circ} \mathrm{C}$, reçoit un inoculum d'une culture de Streptococcus thermophilus TJ (I p. Ioo) et d'une culture de Lactobacillus bulgaricus $\mathrm{LY}_{3}$ ( $\mathrm{I}$ p. Ioo). Ces cultures ont subi en deux jours quatre repiquages successifs à partir de cultures-stocks conservées $\grave{\mathrm{a}}-2 \mathrm{O}^{\circ} \mathrm{C}$.

L'incubation se poursuit durant 4 heures dans un bain-marie à $45^{\circ} \mathrm{C}$. Le $\mathrm{pH}$ du produit obtenu atteint en moyenne $4,95(4,90$ à $5, \mathrm{IO})$. Ce produit est conservé à $4^{\circ} \mathrm{C}$ jusqu'à son utilisation, le lendemain ou le surlendemain de la préparation.

\section{b) Animaux.}

L'expérience porte sur 59 rats Wistar mâles pesant environ $25^{\circ} \mathrm{g}$, recevant depuis le sevrage la même alimentation (biscuits "Extralabo »).

\section{c) Déroulement de l'expérience.}

Les animaux sont préparés par une technique s'inspirant de celle de Peraino et al. (r959) pour que le degré de vacuité du tube digestif soit sensiblement le même chez tous les rats, on les entraîne à consommer chaque jour leur ration complète en 4 heures (de $8 \mathrm{~h} 30$ à I $2 \mathrm{~h} 30$ ); pendant le reste de la journée, ils ne reçoivent que de l'eau. L'entraînement ne demande que 8 jours lorsque les animaux sont placés à l'obscurité pendant la période d'alimentation et à la lumière de $12 \mathrm{~h} 30$ à $8 \mathrm{~h} 30$ le lendemain. Pendant les 3 premiers jours, ils se sous-alimentent et perdent du poids; mais dès le huitième jour. ils sont parfaitement accoutumés au rythme d'alimentation imposé et ont retrouvé leur poicls initial.

Les rats sont alors soumis à un dernier jeûne, l'eau de boisson leur étant retirée à I 8 heures Le lendemain, à $8 \mathrm{~h} 30$, huit d'entre eux sont sacrifiés par décapitation, tandis que les autres reçoivent par intubation $3 \mathrm{ml}$ de lait ou de yogourt (soit $420 \mathrm{mg}$ de matière sèche) portés à $20^{\circ} \mathrm{C}$. Les rats sont répartis en groupes de 4 ou 5 sujets qu'on sacrifie 3 minutes, 30 minutes, 1 heure,2 heures, 4 heures et 8 heures après intubation.

Le prélèvement du contenu stomacal et la détermination du taux de la matière sèche sont réalisés de la même manière que dans l'expérience préliminaire.

(1) L'étude sur animaux ayant été répartie sur plusieurs semainses, l'utilisation des mêmes poudres pour préparer tous les échantillons de lait (et de yoghourt) a paru préférable à l'utilisation de lait frais.

(2) Traitement thermique appliqué dans l'industrie du lait destiné à la préparation du yoghourt. Son but est de détruire en forte proportion la flore bactérienne banale avant l'ensemencement. 


\section{Résultats}

D'après le tableau 3, les quantités de matière sèche trouvées dans l'estomac à tous les stades de la digestion sont légèrement plus élevées chez les sujets qui ont reçu du yogourt que chez ceux qui ont reçu du lait. Mais, compte tenu du petit nombre de valeurs individuelles correspondant à chaque point de la courbe, les différences observées ne sont pas significatives (comparaison par letest du $t$ de Student).

\section{TABLEAU 3}

Teneur du contenu de l'estomac après intubation de $3 \mathrm{ml}$ de lait ou de yoghourt correspondant à $419 \mathrm{mg}$ de matière sèche

(en $\mathrm{mg}$ de matière sèche)

\begin{tabular}{|c|c|c|}
\hline \multirow{2}{*}{ Temps } & \multicolumn{2}{|c|}{$\begin{array}{l}\text { Matière sèche du contenu de l'estomac après intubation } \\
\text { (Moyennes suivies de l'erreur standard de la moyenne) }\end{array}$} \\
\hline & de lait & de yoghourt \\
\hline Avant intubation & \multicolumn{2}{|c|}{$13,4 \pm 1,2$} \\
\hline $\begin{array}{r}3 \text { minutes } \ldots \ldots \ldots \ldots \\
30 \text { minutes } \ldots \ldots \ldots \ldots \\
60 \text { minutes } \ldots \ldots \ldots \ldots \ldots \\
120 \text { minutes } \ldots \ldots \ldots \ldots \\
4 \text { heures } \ldots \ldots \ldots \ldots \ldots \\
8 \text { heures } \ldots \ldots \ldots \ldots \ldots\end{array}$ & $\begin{array}{r}314,0 \pm 25,2 \\
213,8 \pm 29,8 \\
170,0 \pm 23,5 \\
74,8 \pm 19,1 \\
17,2 \pm 1,8 \\
44,4 \pm 15,2\end{array}$ & $\begin{array}{r}362,8 \pm 27,2 \\
220,1 \pm 12,1 \\
178,6 \pm 3,0 \\
92,0 \pm 25,0 \\
23,4 \pm 3,7 \\
28,0 \pm 1,5\end{array}$ \\
\hline
\end{tabular}

Il est difficile de dire si la quantité de matière sèche présente dans l'estomac diminue exponentiellement au cours du temps.

\section{ÉTUDE DE L'ÉVACUA'TION DE L'ESTOMAC APRÈS INGESTION DE LAIT OU DE YOGHOUR'T}

\section{Protocole expérimental}

a) Caractéristiques des produits étudiés.

Le lait et le yogourt sont obtenus de la même manière que dans l'expérience précédente.

Le $\mathrm{pH}$ du lait au moment de l'administration est en moyenne de $6,6 \circ(6,55$ à 6,65$)$ et celui du yogourt de $4,95(4,90$ à 5,00$)$.

b) Animaux.

L'expérience porte sur 124 rats Wistar mâles pesant environ $270 \mathrm{~g}$, recevant depuis le sevrage des biscuits " Extralabo ».

c) Déroulement de l'expérience.

Les animaux sont soumis à un entrainement qui vise à leur faire consommer chaque jour, dans un délai de 4 heures $(8 \mathrm{~h} 30$ à I $2 \mathrm{~h} 30)$ :

— d'abord $3 \mathrm{ml}$ de lait ou de yoghourt; 
- puis, lorsque ces $3 \mathrm{ml}$ sont entièrement consommés, le complément de leur ration sous forme de biscuits (ad libitum).

Pour que le lait et le yoghourt soient ingérés en peu de temps, l'accès à l'eau de boisson est supprimé pendant les 14 heures qui précèdent la distribution des deux produits (de $\mathrm{I} 8 \mathrm{~h} \quad 30$ au lendemain $8 \mathrm{~h} 30$ ).

L'entraînement exige une quinzaine de jours. Pendant les 3 premiers, les rats se sous-alimentent et perdent du poids. Ensuite, ils prennent progressivement l'habitude de consommer le lait et le yoghourt en quelques minutes, s'accoutument au rythme d'alimentation imposé et retrouvent leur poids initial.

Le jour du sacrifice, certains sujets sont décapités après un dernier jeûne, tandis que les autres reçoivent leur ration habituelle de lait ou de yogourt (soit environ $420 \mathrm{mg}$ de matière sèche) et sont sactifiés après 5 minutes, 30 minutes, I heure, 2 heures, 3 heures, 4 heures (délais calculés en considérant le moment de la distribution du lait ou du yoghourt comme "instant zéro ").

\section{Résultats}

D'après le tableau 4, l'évacuation du lait est d'abord plus rapide que celle du yogourt : si bien que, durant I h et demie environ, c'est dans l'estomac des sujets ayant consommé du yogourt qu'on trouve la plus grande quantité de matière sèche. Ensuite, le phénomène s'inverse (prélèvement après 2 heures), mais les deux

\section{TABIEAU 4}

Teneur du contenu de l'estomac après ingestion spontanée de $3 \mathrm{ml}$ de lait ou de yogourt correspondant à $422 \mathrm{mg}$ de $M$. S.

(en mg de matière sèche)

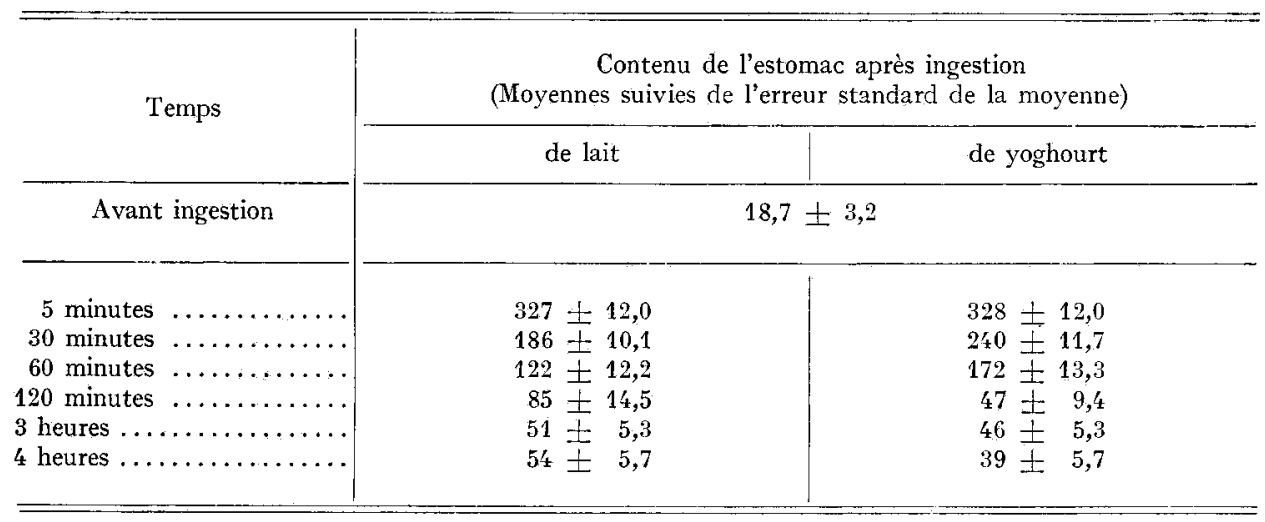

courbes se rejoignent finalement (prélèvement après 3 heures). Les différences entre les deux groupes d'animaux comparées par le test du $t$ de Student, sont:

- non significatives pour la $I^{\text {re }}$ détermination ( 5 minutes) et pour les deux dernières $(3 \mathrm{~h}$ et $4 \mathrm{~h})$;

- hautement significatives pour toutes les déterminations faites entre $30 \mathrm{mi}$ nutes et 2 heures (au seuil de $\mathrm{P}=0$,or après 30 minutes, de $\mathrm{P}=0,02$ après $\mathrm{I}$ heure et après 2 heures).

Ces différences sont plus faciles à interpréter lorsque l'évolution du phénomène est représentée en coordonnées semi-logarithmiques (fig. 2). Pendant les 3 premières heures de la digestion, il existe pour le lait une corrélation négative très élevée entre la quantité de matière sèche présente dans l'estomac et le logarithme du temps 
écoulé depuis l'ingestion. L'équation de régression est : $y=448,3-\mathrm{I} 77,4 \log t$ où $y$ représente la quantité de matière sèche en $\mathrm{mg}$, au temps $t$ en $\mathrm{mn}$, avec $r=-0,998$ (significatif au seuil de $\mathrm{P}=\mathrm{o}, \mathrm{OI}$ ). L'ordonnée à l'origine de cette droite (soit 448) correspond sensiblement à la quantité de matière sèche présente dans l'estomac aussitôt après l'ingestion (20 $\mathrm{mg}$ de matière sèche résiduelle après 20 heures de jeûne $+422 \mathrm{mg}$ reçus sous forme de lait ou de yoghourt) (1).

Quant à l'évacuation stomacale du yoghourt, elle ne s'exprime pas par une loi simple.

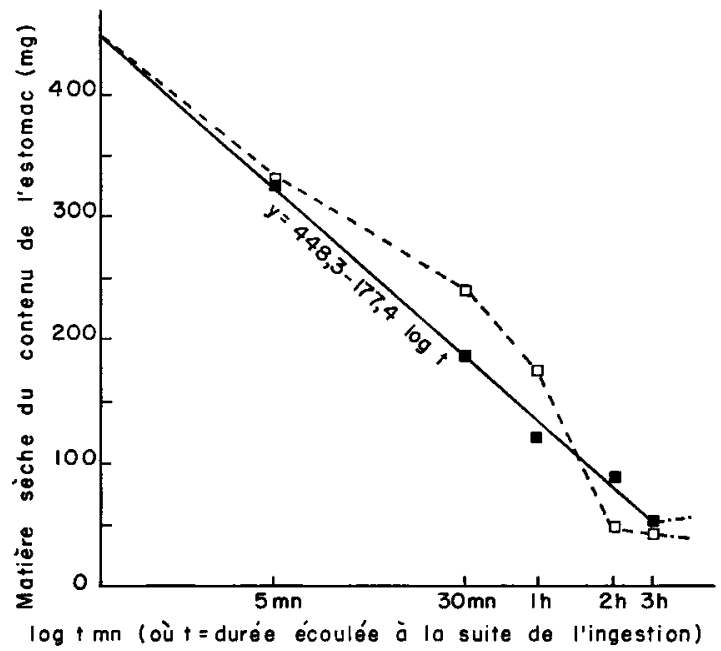

FIG. 2. - Évacuation stomacale de la matière sèche après ingestion de $3 \mathrm{ml}$ de lait ou de yoghourl (représentation en coordonnées semi-logarithmiques)

En trait plein : lait. - En trait discontinu : yoghourt.

\section{DISCUSSION}

I Le lait introduit dans 1'estomac par intubation ou par ingestion spontanée en est évacué avec une rapidité remarquable.

Il ne semble pas que ce phènomène - déjà observé par BECCARI (I957) chez le Cobaye - résulte d'une poussée excessive du contenu stomacal sur le pylore, due elle-même à l'état de réplétion de l'estomac : ce dernier peut contenir une quantité de liquide bien supérieure à la quantité de lait administrée, sans être anormalement distendu. Il est plus probable que le lait se comporte à la manière de l'eau. qui franchit le pylore presque instantanément.

$2^{0}$ Pendant les deux premières heures, les quantités de matière sèche retrouvées dans l'estomac sont un peu plus élevées chez les animauc qui ont reçu du lait par intubation que chez ceux qui l'ont ingéré normalement. Toutefois, les différences ne sont pas significatives, en raison de la forte dispersion des valeurs obtenues dans le cas de l'intubation, et du petit nombre de sujets intubés.

(1) Le raisonnement n'est cependant pas tout à fait justifié, puisque l'ingestion du lait et du yogourt n'est pas instantanée. 
De toute manière, nous ne retrouvons pas les résultats de VOROBIEW et KousHENKO (I935) : chez le Chien porteur d'une fistule gastrique, ces derniers observaient que l'évacuation pylorique du lait administré par intubation est plus rapide que celle du lait ingéré Ils attribuaient cette différence au fait que, dans le cas de l'intubation, l'absence des réflexes dus au lapage et à la succion entraîne une diminution de la quantité de suc gastrique sécrétée.

$3^{0}$ Nos observations sont en désaccord avec l'affirmation classique selon laquelle les laits fermentés quitteraient l'estomac plus rapidement que le lait (1). Mais elles s'accordent avec celles de BALzol, et al. (I96I) qui, par radiographie, observent chez l'Homme une évacuation plus lente du yoghourt pendant les deux premières heures.

Il n'est pas facile d'interpréter les différences observées, dans le présent travail, entre l'évacuation stomacale du yoghourt et du lait chez les animaux qui ont ingéré ces produits $\left({ }^{2}\right)$.

Bien qu'il ne soit pas aussi fluide que le lait, le yoghourt distribué aux animaux peut encore être considéré comme un liquide : il franchit le pylore aussi vite que le lait pendant les premières minutes.

Ensuite, le transit des deux produits se différencie : dans une première phase, le yoghourt est évacué plus lentement que le lait; puis les deux produits quittent l'estomac sensiblement à la même vitesse. Dans une dernière phase, le phénomène s'inverse et le yoghourt est évacué plus rapidement que le lait. Finalement, à considérer le phénomène dans son ensemble, il apparaîtrait que le yoghourt quitte l'estomac plus rapidement que le lait, alors qu'en réalité les courbes d'évacuation sont très dissemblables.

La connaissance de l'évolution du $\mathrm{pH}$ du contenu stomacal au cours de la digestion des deux produits aiderait peut-être à interpréter ces résultats. En effet, selon les conceptions anciennes de CANNON l'ouverture du sphincter pylorique serait commandée par l'acidité du chyme, et sa fermeture par le contact même du chyme acide avec la muqueuse duodénale : toutefois ces conceptions sont aujourd'hui très combattues. D'autre part, on peut se demander si le $\mathrm{pH}$ n'influe pas sur la qualité du coagulum, qui doit jouer elle-même dans l'évacuation de ce dernier.

Dans une nouvelle série d'expériences, nous avons donc l'intention de suivre l'évolution du $\mathrm{pH}$ du contenu stomacal au cours de la digestion du lait et du yoghourt.

$4^{\circ}$ L'étude de $1^{\prime}$ évacuation stomacale de la matière sèche des aliments ne donne qu'une information d'ensemble sur leur transit dans l'estomac, puisque les différents constituants de la matière sèche ne sont pas toujours évacués simultanément.

Dans le cas présent, on peut se demander si la matière sèche évacuée dès les premières minutes ne serait pas constituée en majeure partie par les constituants en solution dans le lait, - comme le lactose et les lactoprotéines -, la caséine et les matières grasses englobées dans le coagulum quittant l'estomac plus tardivement.

Il serait intéressant en particulier de déterminer la valeur du rapport azote/matière sèche du contenu stomacal aux divers stades de la digestion du lait et du yoghourt.

(1) Voir à ce sujet l'introduction.

(2) Il ne sera question ici que de la comparaison lait-yoghourt après ingestion. Dans le cas de l'intubation, on a vu que les différences observées entre le transit des deux produits sont peu marqués et non significatives. 
$5^{\circ}$ Dans l'état actuel des connaissances, nous ne saurions dire dans quelle mesure la rapidité avec laquelle le lait introduit dans un estomac en état de vacuité en est évacué, constitue pour l'organisme un avantage, et dans quelle mesure elle entraîne pour lui des inconvénients. Nous ne saurions dire non plus si les particularités propres au transit du lait et à celưi du yogourt peuvent être invoquées en faveur de l'un ou l'autre des deux produits.

Chez l'Homme, la brièveté du séjour des aliments dans l'estomac pourrait favoriser en particulier l'activité dans la période qui suit les repas $\left({ }^{1}\right)$ : cette considération pratique n'est pas sans intérêt, à une époque où la " journée continue " - n'impliquant qu'une brève interruption dans la journée de travail - tend à se développer.

Mais, d'autre part, il ne faut pas sous-estimer l'importance de l'étape gastrique dans les processus digestifs. Dans le cas présent, si la matière sèche évacuée dès les premières minutes dans le duodénum est formée principalement de lactose et d'acide lactique, la digestion du lait et du yoghourt ne peut en être affectée défavorablement. En revanche, si elle comprend aussi une forte proportion de protéines, il devient nécessaire de se demander si ces dernières ont été suffisamment préparées aux étapes ultérieures de leur digestion.

Sur ce point encore, la détermination de l'évacuation du rapport azote/matière sèche du contenu stomacal au cours du temps fournirait d'utiles informations.

Reçu pour publication en janvier 1967.

\section{REMERCIEMEN'TS}

Nous adressons nos vifs remerciements à M. VASSAL, Ingénieur à l'I. N..R. A. dont les conseils nous ont permis de mettre au point les conditions de chauffage et de fermentation du lait.

Nous remercions également les Établissements France-Lait, qui nous ont procuré les échantillons de lait en poudre utilisés dans ce travail.

\section{SUMMARY}

COMPARED STOMACH-EMPTYING OF MILK AND YOGURT IN THE RAT

Adult rats were ingested or stomach-intubated either milk containing I $30 \mathrm{~g}$ of dry matter and $10 \mathrm{~g}$ of fat per liter or yogurt obtained by selected lactic ferments from the same milk. The animals were slaughtered at various intervals after feeding and the amounts of dry matter in the stomachs were estimated.

I. - Gastric emptying of milk is very fast, whatever wary it is given : $25 \mathrm{p}$. I00 of the given amount had emptied from the stomach within Io minutes, $50 \mathrm{p}$. I00 within half an hour, $7^{6} \mathrm{p}$. 100 within $\mathrm{r}$ hour.

The amount of dry matter remaining in the stomach $(y \mathrm{mg})$ is for the first 3 hours a semi-logarithmic function of the time elapsed since feeding ( $t$ minutes) :

$$
y=-\mathrm{r} 77,4 \log t+448
$$

(1) Voir Causeret (1957). 
2. - Gastric emptying of intubated yogurt is slower, but the emptying of ingested yogurt is not a simple function of the time elapsed since ingestion. At first, it was slower than milk emptying : $36 \mathrm{p}$. I00 of the given amount of yogurt dry matter remained in the stomach one hour after ingestion versus $24 \mathrm{p}$. 100 for milk. Then, the rate of emptying increased so that yogurt had totally emptied from the stomach earlier than milk.

\section{RÉFÉRENCES BIBLIOGRAPHIQUES}

Balzola F., Kincsìs A., Demole M., ig6i L'influence d'un coagulant du lait sur l'évacuation gastrique, et son utilisation dans le traitement de l'obésité. Nutr. Dieta, 3, 65-74.

Beccari E., 1957. La velocità di svuotamento dello stomaco nelle cavia. Bol. Soc. ilal. Biol. sper., 33, 523-524.

Causeret J., 1957 . Nutrition et capacités physiques. Bull. Soc. sci. Hyg. aliment., 45, 19-57.

Cornwelt. G. G., KillenserG P. G., 1965. The effect of a low fat, low calorie synthetic nutrient (Metrecal) on gastric secretion and emptying in man. Amer. J. digest. Dis., 10, 22-30.

EBEL D., 1953. Die Magennenteerungszeit von normaler und homogenisierter Kuhmilch im Säuglings und Kindesalter. Z. Kinderheilk, 72, 342-346.

Fetter D., Schlutz F. W., I935. Digestion of milk and of a modified milk in vivo. Amer. J. Dis. Child. 50, IIO7-III2.

GüTZLAFF W., Jochims J., r954. Über Magenverweildaner und Labfähigkeit von Sterilmilch. Z. Kinderheilk., 74, 547-552.

NoORden (C. Von), Salomon H., I920. Handbuch der Ernährungslehre. Allgemeine Diätetik, vol. I, p. 270 et 280 , Springer, Berlin.

Peraino C., Rogers Q. R., Yoshida M., Chen L. M., Harper A. E., 1959. Observations on protein digestion in vivo. Canad. J. Biochem. Physiol., 37, I475-1491.

Somogyi J. C., ig6o. Ernährungsphysiologische Aspekte der Somermilchprodukte. Nutr. Dieia, 2, 39-46.

Tobler L., 1906. Utber Magenverdanung der Milch. Dische Med. Vschr., 32, I645.

VASSAL L., i 964. Étude sur le lait destiné à la fabrication du yoghourt. (communication personnelle).

Vorobiew A. M., Koushenko S. T., i935. Activité sécrétrice et évacuatrice de l'estomac de jeunes chiens dans les différents régimes alimentaires (en russe, résumé français). Med. Exp. Ukr., n⿳0.⿲丿⿺乚一匕 $73-83$. 